\title{
A New Quantitative Method for Studying the Vulnerability of Civil Aviation Network System to Spatially Localized Hazards
}

\author{
Hang $\mathrm{Li}^{1,2,3} \cdot$ Xiao-Bing $\mathrm{Hu}^{1,2,4} \cdot$ Xiaomei Guo ${ }^{1,2} \cdot \mathrm{Zhen}_{\mathrm{Xu}^{1,2}}$. \\ P. H. A. J. M. van Gelder ${ }^{3}$
}

Published online: 21 September 2016

(c) The Author(s) 2016. This article is published with open access at Springerlink.com

\begin{abstract}
As an important infrastructure system, civil aviation network system can be severely affected by natural hazards. Although a natural hazard is usually local, its impact, through the network topology, can become global. Inspired by Wilkinson's work in 2012, this article proposes a new quantitative spatial vulnerability model for network systems, which emphasizes the spreading impact of spatially localized hazards on these systems. This model considers hazard location and area covered by a hazard, and spatially spreading impact of the hazard (including direct impact and indirect impact through network topology) and proposes an absolute spatial vulnerability index and a relative spatial vulnerability index to reflect the vulnerability of a network system to local hazards. The model is then applied to study the spatial vulnerability of the Chinese civil aviation network system. The simulation results show that (1) the proposed model is effective and useful to study spatial vulnerability of civil aviation network systems as the results well explain the general situation of the Chinese civil aviation system; and (2) the Chinese civil aviation network system is highly vulnerable
\end{abstract}

Xiao-Bing $\mathrm{Hu}$

huxiaobing@bnu.edu.cn

1 Academy of Disaster Reduction and Emergency Management, Beijing Normal University, Beijing 100875, China

2 State Key Laboratory of Earth Surface Processes and Resource Ecology, Beijing Normal University, Beijing 100875, China

3 Faculty of Technology, Policy and Management, Delft University of Technology, Delft, The Netherlands

4 School of Engineering, University of Warwick, Coventry, UK to local hazards when indirect impacts through network connections are considered.

Keywords Civil aviation · Network system · Spatially localized hazards · Vulnerability

\section{Introduction}

Civil aviation, as an advanced transportation mode, is not only closely linked with our daily life, but also significantly important for the economic development of countries and regions. According to the 2013 Annual Report of the International Civil Aviation Organization Council (ICAO 2014), the number of world air passengers reached 3.1 billion, air freight (expressed as freight ton-kilometer performed) rose to approximately 49.3 million tons, and net profit of the world air transport reached USD 181 billion in 2013. As an important sector of the economy, civil aviation system has become a crucial infrastructure system supporting the modernization of economies and societies and promoting the development of various other related industries, such as tourism, trade, and logistics. However, in the context of global climate change, the sustainable development of the world civil aviation industry is facing increasingly more severe challenges imposed by frequent natural hazards, especially meteorological hazards. Generally, these hazards are spatially restricted, so we use the term "spatially localized hazards" in this article.

Spatially localized hazards, such as torrential rain, typhoon, snowstorm, and dust storm, may directly result in closure of airports and routes, damage airport and en-route navigation equipment/facilities, cause severe flight delays, and lead to aviation accidents and even catastrophes. For example, the 2010 eruption of the Eyjafjallajokull Volcano 
in Iceland resulted in the closures of European airports and routes at a very large scale, causing more than 10 million passenger delays (Mazzocchi et al. 2010). Civil aviation system, as a network system, may be globally influenced by such spatially localized hazards, as negative effects of the hazards can spread along flight routes between airports in the system. For example, as shown in Fig. 1, assuming a local hazard happens in a simplified civil aviation network system, where Airport 1 is directly affected by the hazard. As Airport 2 has flights from/to Airport 1, it will be disturbed indirectly by the hazard because flights between Airport 2 and Airport 1 may not take off on time or be forced to cancel. Although Airport 3 has no direct flights from/to Airport 1, it may also be impacted indirectly because Airport 3 has flights from/to Airport 2, which are likely to be affected by the delays or cancelation of flights between Airport 1 and Airport 2. Finally, the whole system is affected by the hazard. Therefore, it is important to study the impacts of local hazards on civil aviation network system.

Vulnerability is an important concept to assess the performance of a system in the presence of hazards. At present, studies on the vulnerability of civil aviation network system are concentrated in the field of system science. Researchers mainly apply complex network theories to study the performances and characteristics of different network topologies in the face of hazards (Burghouwt et al. 2003; Chi et al. 2003; Gastner and Newman 2006). Many countries' civil aviation systems have been proved to be scale-free networks, whose degree distribution follows a

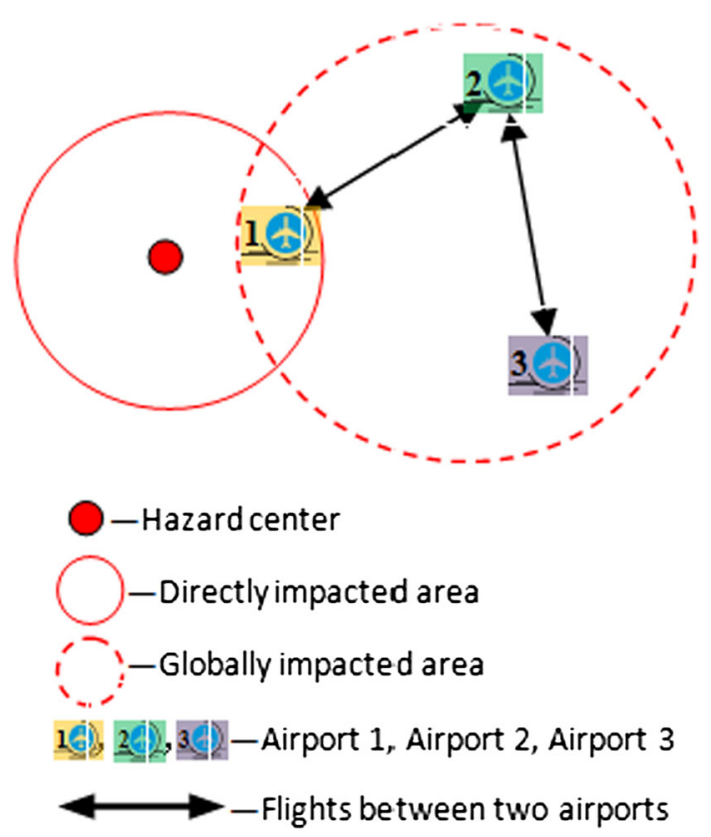

Fig. 1 A local hazard-impact scenario in a simplified civil aviation network system power law and therefore comprises a small number of highdegree nodes, that is, hub nodes, and a large number of low-degree nodes, that is, spoke nodes (Guimera and Amaral 2004; Li and Cai 2004). Such networks have been shown to be resilient to random hazards but are vulnerable to intended attacks, because when compared with an intended attack especially targeting at hub nodes, a random hazard has a much smaller possibility to cover and affect a hub node (Albert et al. 2000). Most of the existing studies mainly focus on network topology, largely ignoring the spatial distribution of hazards, as well as the spreading impact of spatially localized hazards, which may have increasingly adverse impact on civil aviation systems under the background of global climate change. Some researchers have considered the impact of spatial hazards when studying vulnerability of civil aviation networks. Wilkinson et al. (2012) qualitatively analyzed the spatial vulnerability of the European air traffic network after the 2010 eruption of the Eyjafjallajokull Volcano. Janić (2015) developed an integrated model to analyze the resilience, friability, and costs of the northeast part of the US air transport network affected by Hurricane Sandy in October 2012. Our research further proposes a quantitative method to study the spatial vulnerability of civil aviation network systems affected by local hazards.

In Sect. 2, a new quantitative spatial vulnerability model is introduced to assess the performance of network systems in the presence of spatially localized hazards from the viewpoint of disaster science. This model simultaneously considers characteristics of spatially localized hazards and topologies of network systems, and emphasizes on the spreading impact of the hazards. Based on the model, we carry out a case study on the spatial vulnerability of the Chinese civil aviation network system, and report some simulation results in Sect. 3. The main conclusions are presented in Sect. 4.

\section{A Quantitative Spatial Vulnerability Model for Network Systems}

When defining vulnerability of a system (IPCC 2001; Cutter et al. 2003; Turner et al. 2003; Shi et al. 2009), researchers have paid little attention to the spreading impact of local hazards transmitted through networks. Although Wilkinson et al. (2012) has made an attempt to analyze the spatial vulnerability of the European air traffic network, the study lacks explicit definition and a quantitative method for spatial vulnerability analysis of network systems. In response, our study first defines the spatial vulnerability of a network system as the likelihood of a given system as a whole to be harmed from exposure to local hazards, such as rainstorms and earthquakes. Then, 
the model proposed in this article focuses on how to mathematically formulate and quantify the spatial vulnerability of a network system.

\subsection{Basic Idea}

In various fields of system engineering, researchers have developed many methods to analyze the vulnerability of network systems, including maximal flow (Wollmer 1964; Ghare et al. 1971; Wood 1993), shortest path (Corley and Sha 1982; Israeli and Wood 2002; Lim and Smith 2007), connectivity (Grubesic et al. 2003; Murray and Grubesic 2007; Arulselvan et al. 2009), system flow (Myung and Kim 2004; Murray et al. 2007), access fortification (Church et al. 2004; Church and Scaparra 2007), and component attributes approach (Grubesic and Murray 2006). Basically, these methods aim to find components in a network that, if removed or rendered inoperable, would influence the system performance most significantly. Network topology is usually the focus of such vulnerability analysis methods. Hazard characteristics, although prominent in vulnerability research in the disaster risk field (where dose-response analysis is a well-known method) ( $\mathrm{Li}$ et al. 2008; Ding and Miao 2014), are largely overlooked. Dose-response methods are useful for assessing the vulnerability of individual units in a network system (Tran et al. 2010; Zhou et al. 2014), but they often fail to consider the spreading impact and/or cascading effect of spatially localized hazards on a system because of network topology.

Our proposed model is based on the traditional vulnerability curve method. The basic idea of traditional vulnerability curve is to build a functional relationship between hazard intensity and hazard impact for a specific hazard category (Jaiswal et al. 2011; Omidvar et al. 2012; Papathoma-Köhle et al. 2012). Figure 2 presents an example of such method. This vulnerability curve can illustrate how a system performs under a given hazard.

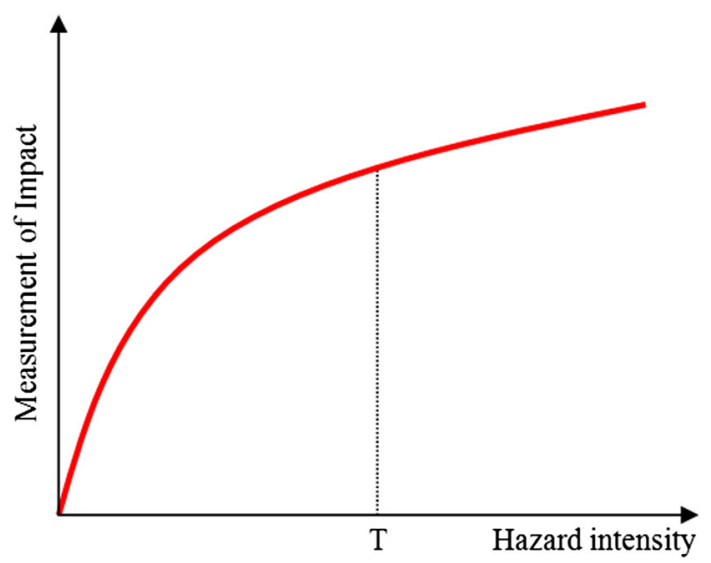

Fig. 2 Traditional vulnerability curve model
Here, system performance is used in a general sense, which may refer to certain function or property of a system to be likely affected by hazards. That is, the vulnerability curve depicts to what extent a system may be affected by hazards in terms of certain function or property with which we are most concerned. However, the curve cannot tell how the system's vulnerability is related to the location of a hazard, size of the hazard covered area, and direct and indirect impacts of the hazard. For a given hazard category with a specific hazard intensity, different hazard locations may lead to different impacts on a system. For example, a rainstorm in an area with dense airports usually has a more severe impact on an aviation system than that happens in an area with few airports, because more flights could be cancelled or delayed due to the rainstorm occurred in the area with more airports. For a given hazard intensity, different sizes of area covered by hazard may also result in different impacts on a system. Whether to consider indirect impacts of hazards through network connections will also make a great difference in assessing the vulnerability of a network system.

According to the definition of spatial vulnerability in this article, two basic factors are important to calculate the spatial vulnerability of a network system: area covered by hazards (multiple hazards may occur simultaneously in different locations in a network system), and spatially spreading impact of hazards (including direct impact and indirect impact of hazards). Then, for a given hazard intensity, we can establish a mathematical relationship between these two factors, and therefore get an impact curve between area covered by the multi-hazards and spreading impact of hazards. Hazards at different locations may have greatly different influences on a system, even with the same hazard covered area. More descriptions are introduced in Sect. 2.3. Note that this new impact curve is different from the traditional vulnerability curve, which is between hazard intensity and impact of hazard (Fig. 2). In addition to the new impact curve, we further introduce a concept of "neutral line," in order to analyze spatial vulnerability in a relative term.

\subsection{Concept of Neutral Line}

The concept of neutral line can be explained by examining the following two questions.

Question 1 After a rainstorm with a return period of 100 years, a megacity with a GDP of USD 10 billion per year recorded a loss of USD 10,000. Is this city vulnerable to rainstorms?

Question 2 After an earthquake, a modern skyscraper recorded a loss of USD 1 million because of damages to its interior decorations, while a very small historical building recorded a loss of USD 0.1 million because it was 
completely destroyed. Which building is more vulnerable to earthquake?

If vulnerability is measured by absolute loss terms or the impact curve, then the city in question 1 will be considered vulnerable despite the negligible loss caused by the rainstorm compared to the total GDP of the city, and the skyscraper in question 2 will be considered more vulnerable than the small historical building. However, our common sense would suggest that the city in question 1 is not vulnerable, and the skyscraper in question 2 is less vulnerable than the historical building. Therefore, an impact curve is not sufficient for describing vulnerability.

In this article, we describe the spatial vulnerability of a network system using not only an impact curve but also a neutral line. Simply speaking, a neutral line reflects certain expectation on the resistance of a network system against spatially localized hazards. In other words, according to certain knowledge (for example, common sense or theoretical analysis) about the resistance of a system, we would expect/predict the system to record a loss of certain level under a given hazard scenario. A neutral line is defined based on such an expected level of loss. If the actual loss caused by a hazard is below the corresponding expected level given in the neutral line, it means the actual loss is expected according to our priori knowledge, and therefore, we are less likely to be shocked or terrified by the hazard event. If the actual loss exceeded the expected level, we may be surprised and consider the system vulnerable to the hazard.

By this definition, when the spatial vulnerability of a network system is zero, it does not mean that a hazard has no impact on the system. Zero vulnerability means the impact of a hazard is well expected and can be absorbed/ dissipated by the system, and thus has little surprising harm to the system. For a given hazard intensity and area covered by the hazard, we can define a threshold of impact expectation. Only when the actual impact of the hazard is above the threshold, the system is vulnerable to the hazard. If the actual impact is below the threshold, the system is robust to the hazard. If the actual impact equals to the threshold value, then, the system is neutral to the hazard, that is, the system vulnerability to the hazard is zero. Under a given hazard intensity, for different sizes of area covered by hazards, we have different thresholds of impact expectation correspondingly. As the size of hazard covered area changes, the expected impact as threshold also changes. When the size of the area covered by hazards changes from zero to the whole network system, the curve formed by those associated thresholds forms the neutral line under the given hazard intensity.

A neutral line is a crucial standard against which we can judge whether or not a network system is vulnerable to spatially localized hazards. Therefore, a proper definition of neutral line is very important to study the spatial vulnerability of a network system. As mentioned before, a threshold is a certain expectation on the impact to the system under a given hazard scenario (specific hazard intensity and size of area covered by hazards). Therefore, it can be defined according to problem characteristics or common senses. For simplicity, in this article, hazard impact is expected proportional to the size of area covered by hazards.

For a given hazard intensity, if we have an impact curve and a neutral line, then we may determine the spatial vulnerability of a network system qualitatively.

\subsection{A Qualitative Method on Spatial Vulnerability of a Network System}

As discussed in Sect. 2.2, to assess the spatial vulnerability of a network system under a given hazard intensity, we need to derive two important curves: an impact curve and a neutral line. Wilkinson et al. (2012) has used the spatial impacts of a volcanic ash cloud on European air traffic network as the impact curve and impacts of a random hazards on the network as the neutral line to qualitatively analyze the vulnerability of European air traffic network to spatial hazards. In this article, we introduce a general approach to derive the impact curve and the neutral line for determining the vulnerability of a network system.

This study focuses on the vulnerability of network systems, which are associated with certain spatial areas. A hazard may cover any part of the area associated with a network system. For example, in the case of the Chinese domestic civil aviation network, the territory of China is the spatial area of the system, and a rainstorm may only cover a few provinces in China. We divide the size of the hazard covered area by the size of the entire network system area, and obtain the percentage of area covered by the hazards. Hereafter, we define the impact curve by measuring the spreading impact of hazards on the network system against the percentage of area covered by hazards.

The definition of spreading impact of hazards on the network system is highly problem dependent. For example, we can use the number of impacted nodes in the network system to quantify the impact of hazards. We can also use the volume of function losses/failures in the network system to calculate the spreading impact of hazards. In this section of mathematical description, we simply use the percentage of effectively impacted nodes in the network system to define the spreading impact of hazards on the network system.

For a network system, if we know the size of an area covered by hazards, that is, the percentage of hazard covered area, then we can analyze the impact of hazards on the system in terms of the percentage of effectively impacted 
nodes. For example, in Fig. 3b, assuming that three hazards, hazard 1 , hazard 2 , and hazard 3 happen simultaneously in a simplified network system that contains 20 nodes. The area covered by the hazards is $40 \%$ of the total area of the network system, and 14 nodes of the system are effectively affected by the hazards, that is, the percentage of impacted nodes is $70 \%$. If we move hazard 3 to the bottom-left side of Fig. 3b, although the total size of areas covered by the hazards remains the same, the percentage of effectively impacted nodes decreases to $40 \%$. This illustrates that different hazard locations may have different impacts on a system-even if the total size of areas covered by the hazards stays the same, the impact of hazards on the system as a whole may vary greatly, depending on the locations of the hazards.

Therefore, we need to further introduce the concept of average percentage of effectively impacted nodes under a specific percentage of hazard covered area. That is, in a network system of fixed nodes distribution and for a given percentage of hazard covered area, we need to perform hazard impact simulations for many times by changing hazard locations, then calculate the average percentage of effectively impacted nodes, to derive the hazard impact value under this specific hazard covered area.

When the percentage of hazard covered area changes from 0 to $100 \%$, for each specific percentage of hazard covered area, an average percentage of effectively impacted nodes will result from these simulations. An impact curve can be plotted by these average percentages of effectively impacted nodes against the percentages of hazard covered area (Fig. 3a).

Next, we mathematically define the neutral line. In this article, for simplicity, a reasonable expectation on the resistance of a network system against hazards is that the average percentage of effectively impacted nodes should equal to the percentage of hazard covered area. Therefore, the neutral line should be defined as a $45^{\circ}$ straight line from the point $(0 \%, 0 \%)$ to the point $(100 \%, 100 \%)$ (the blue line in Fig. 3a). According to the impact curve and the neutral line, we can qualitatively determine whether a network system is vulnerable to spatially localized hazards. If the impact curve completely overlaps with the neutral line, the network system is neutral to the hazards. If the impact curve is mainly above the neutral line, the system is vulnerable to the hazards (the red line in Fig. 3a). If the impact curve is mainly below the neutral line, the system is robust to the hazards (the green line in Fig. 3a).

An impact curve against a neutral line as in Fig. 3a may illustrate how a network system performs in the face of spatially localized hazards, but it is still necessary to develop a quantitative method to calculate the spatial vulnerability of a network system.

\subsection{A Quantitative Method for Spatial Vulnerability of a Network System}

To quantify spatial vulnerability of a network system, based on the impact curve and neutral line concepts, we define a spatial vulnerability index (SVI). Preliminary conceptualizations have been reported in $\mathrm{Li}$ et al. (2014, 2015). For a given network system, if its SVI is positive, then the system is vulnerable to spatially localized hazards; if its SVI is zero, then the system is neutral to spatially localized hazards; if the SVI is negative, then the system is robust to spatially localized hazards. In the following, we define two specific SVIs: absolute spatial vulnerability index (ASVI) and relative spatial vulnerability index (RSVI).

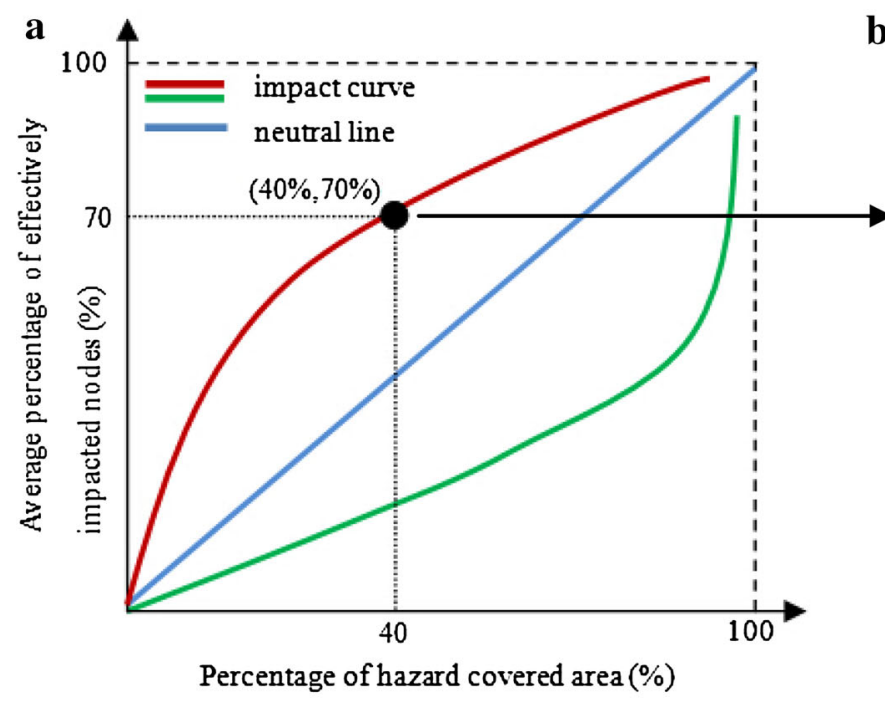

b

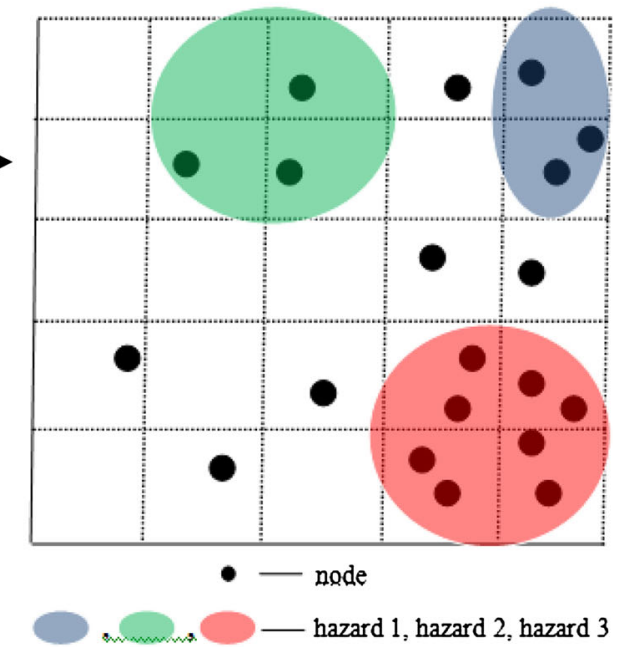

Fig. 3 Impact curve and neutral line (a) and calculation for impact of hazards with a specific hazard covered area (b) 
(1) Absolute spatial vulnerability index (ASVI)

The ASVI is calculated as the integral value of the difference between the impact curve and the neutral line in Fig. $3 \mathrm{a}$ as the percentage of hazard covered area changes from 0 to $100 \%$. The mathematical description of ASVI is

$V_{\mathrm{ASVI}}=\int_{0}^{1}\left(g(x)-g_{\mathrm{NL}}(x)\right) d x$

where $x$ is the percentage of hazard covered area, $g(x)$ is the average percentage of effectively impacted nodes under a given $x$ value, and $g_{\mathrm{NL}}(x)$ is the associated neutral line value. According to the definition of the neutral line in Sect. 2.3, this study employs

$g_{\mathrm{NL}}(x)=x$

As a quantified spatial vulnerability measurement, the ASVI has more advantages than the illustrative plot in Fig. 3a for analyzing the performance of a network system in the face of spatially localized hazards. For example, in a more general case, as illustrated in Fig. 4, the impact curve (in red) may be above the neutral line in certain $x$ range, whilst below the neutral line in other $x$ range. In such cases, an illustrative plot of impact curve and neutral line can hardly help to draw a conclusion regarding the spatial vulnerability of a network system. The ASVI can help determine whether a network system is vulnerable, neutral, or robust to spatially localized hazards. In other words, if the ASVI is positive/zero/negative, then the system is vulnerable/neutral/robust to spatially localized hazards. Furthermore, the ASVI also makes it possible to quantitatively compare the spatial vulnerability of different network systems, because the value of ASVI can indicate to what extent a network system is vulnerable/ neutral/robust. Basically, a higher positive value of ASVI means more vulnerable, whilst a lower negative value of ASVI means more robust.

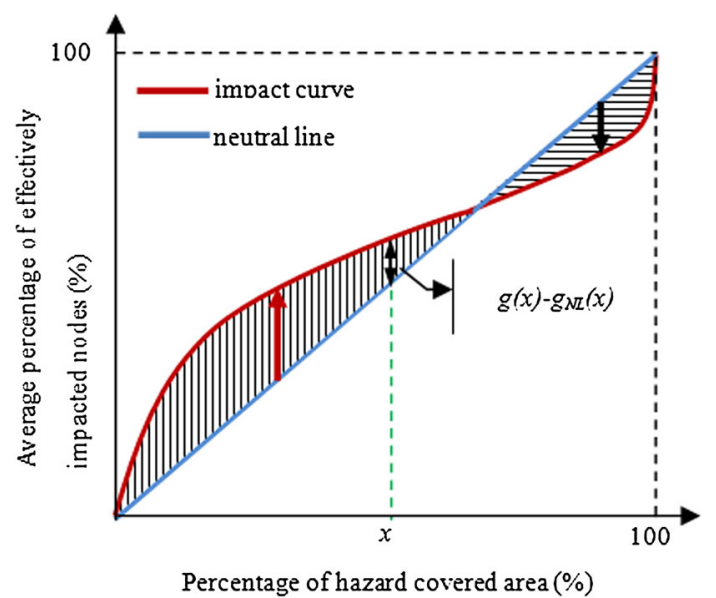

Fig. 4 Absolute spatial vulnerability index (ASVI)
(2) Relative spatial vulnerability index (RSVI)

The ASVI may quantitatively answer question 1 in Sect. 2.2, that is, whether a system is vulnerable or not, but may not be able to address question 2 in Sect. 2.2, that is, to compare two systems. For another example, the ASVI cannot distinguish the vulnerability of the system in Fig. 4 from that of the system in Fig. 5 under a same hazard scenario, as these two systems have the same ASVI value. However, a large network system in Fig. 4 is more vulnerable to spatially localized hazards because of the higher likelihood of occurrence of small-scale hazards with relatively low percentage of hazard covered areas. Therefore, we define a RSVI as follows.

$V_{\mathrm{RSVI}}=\int_{0}^{1} \frac{g(x)-g_{\mathrm{NL}}(x)}{g_{\mathrm{NL}}(x)} d x$

For a same value of $g(x)-g_{\mathrm{NL}}(x)$, small-scale hazards have a smaller $g_{\mathrm{NL}}(x)$ value than large-scale hazards. As a result, the system in Fig. 4 has a larger RSVI than the system in Fig. 5. This indicates that the system in Fig. 4 is more vulnerable to spatially localized hazards than the system in Fig. 5.

\section{A Case Study}

We apply the proposed spatial vulnerability model to study the Chinese domestic civil aviation network system. In this case, network nodes refer to airports and links between nodes refer to direct flights between airports. To comprehensively analyze the spatial vulnerability of the Chinese civil aviation network system to local hazards, we use three hazard scenarios. In scenario 1 , the occurrence probability of hazards is assumed equal everywhere in the whole country, that is, hazards follow a uniform spatial distribution. In scenario 2 and scenario 3 , the distribution of

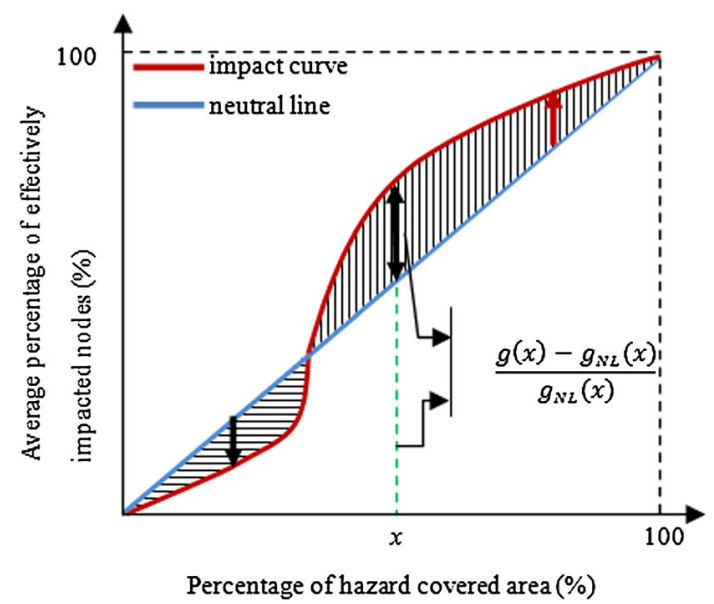

Fig. 5 Relative spatial vulnerability index (RSVI) 
hazards in China is spatially uneven. In scenario 2, we determine the occurrence probability of hazards in different areas based on the spatial distribution of rainstorms in China. In scenario 3, we derive the occurrence probability of hazards according to the spatial distribution of sandstorms in China. For each hazard scenario, we set up three test cases with different considerations of impact:

Test case 1 Only consider the airports located in the area covered by the hazards;

Test case 2 Also take into account airports that have direct flights from/to any airport located in the area covered by the hazards;

Test case 3 Only consider the daily passenger capacity of airports located in the area covered by the hazards. The daily passenger capacity of an airport is the sum of aircraft capacity of all flights from/to the airport during an operational day.

\subsection{Data}

From the largest Chinese travel website Qunar (2014), we collected the flight data of 174 Chinese civil airports, which are taken as the nodes of the network (Figs. 6,7). The connections of network are set up based on the flights between these 174 airports in the period from 1 to 30 April 2014. The daily passenger capacity of an airport is the average result based on all flights from/to the airport during the 30 day period. The daily passenger capacity between a pair of airports is the sum of aircraft capacity of all flights between the two airports. We used the data of flights and passenger capacities to set up an airportlink matrix and a passenger-capacity matrix.

The distribution of rainstorms and sandstorms is obtained as follows: we first collected the data on the occurrences of rainstorms and sandstorms at or near the airport sites between 2004 and 2013 from the Chinese Meteorological Data Services (Chinese Meteorological Data Services n.d.). Then, using the ArcGIS software and the inverse distance weighted method (IDW), the spatial distribution surfaces of rainstorms and sandstorms were generated (Figs. 6, 7). In the next step, we use the Jenks method in ArcGIS to divide the spatial distribution of rainstorms and sandstorms into four levels: low, relatively low, relatively high, and high (Figs. 6, 7). These four levels are used to determine the hazard occurrence probability in a given area. For example, in the hazard simulation, if an area has a low/relatively low/relatively high/high level of rainstorms, then the probability for a rainstorm to happen in this area is $0.2 / 0.4 / 0.6 / 0.8$.

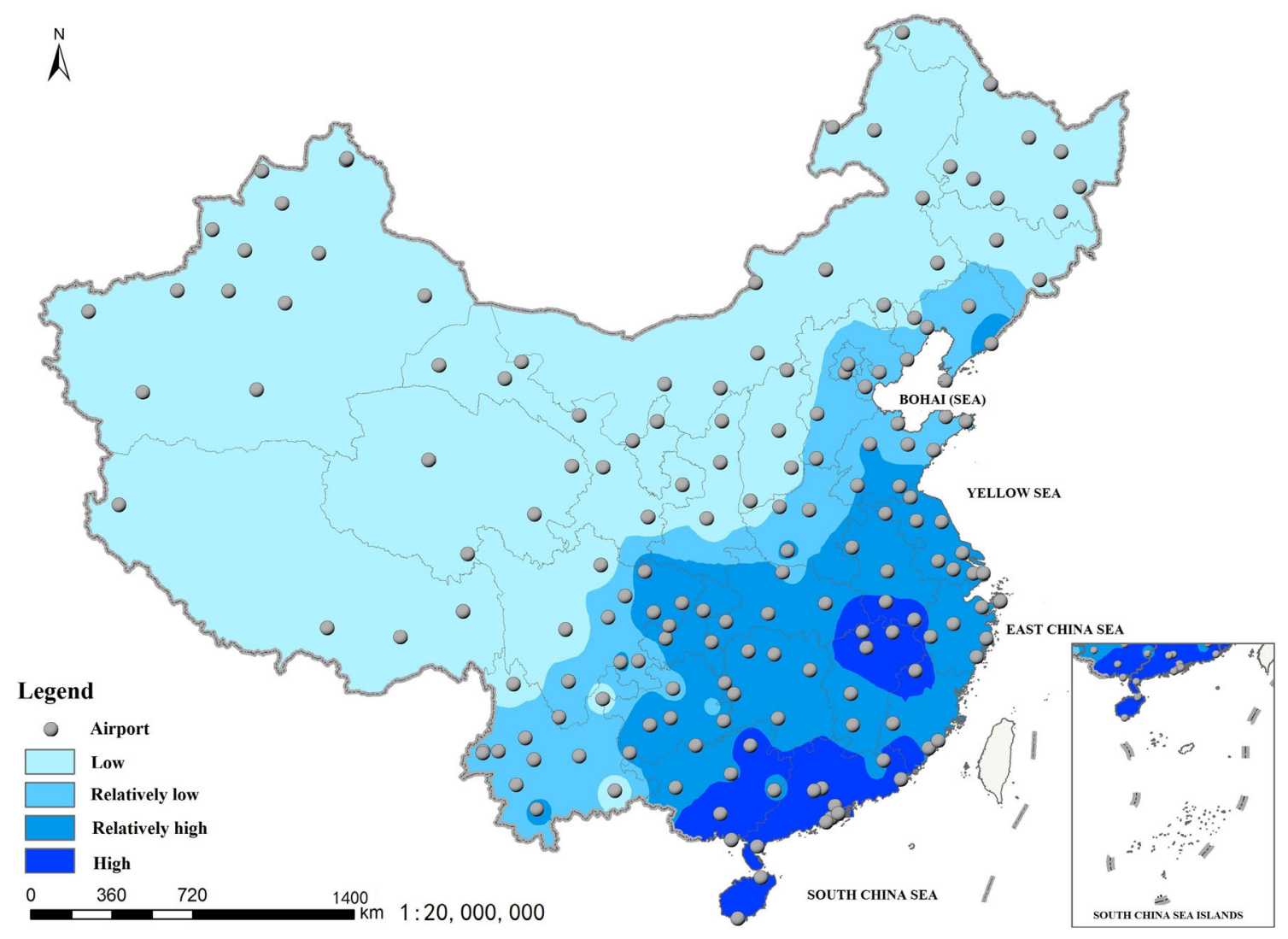

Fig. 6 Spatial distribution of Chinese civil airports and rainstorm occurrence frequencies 


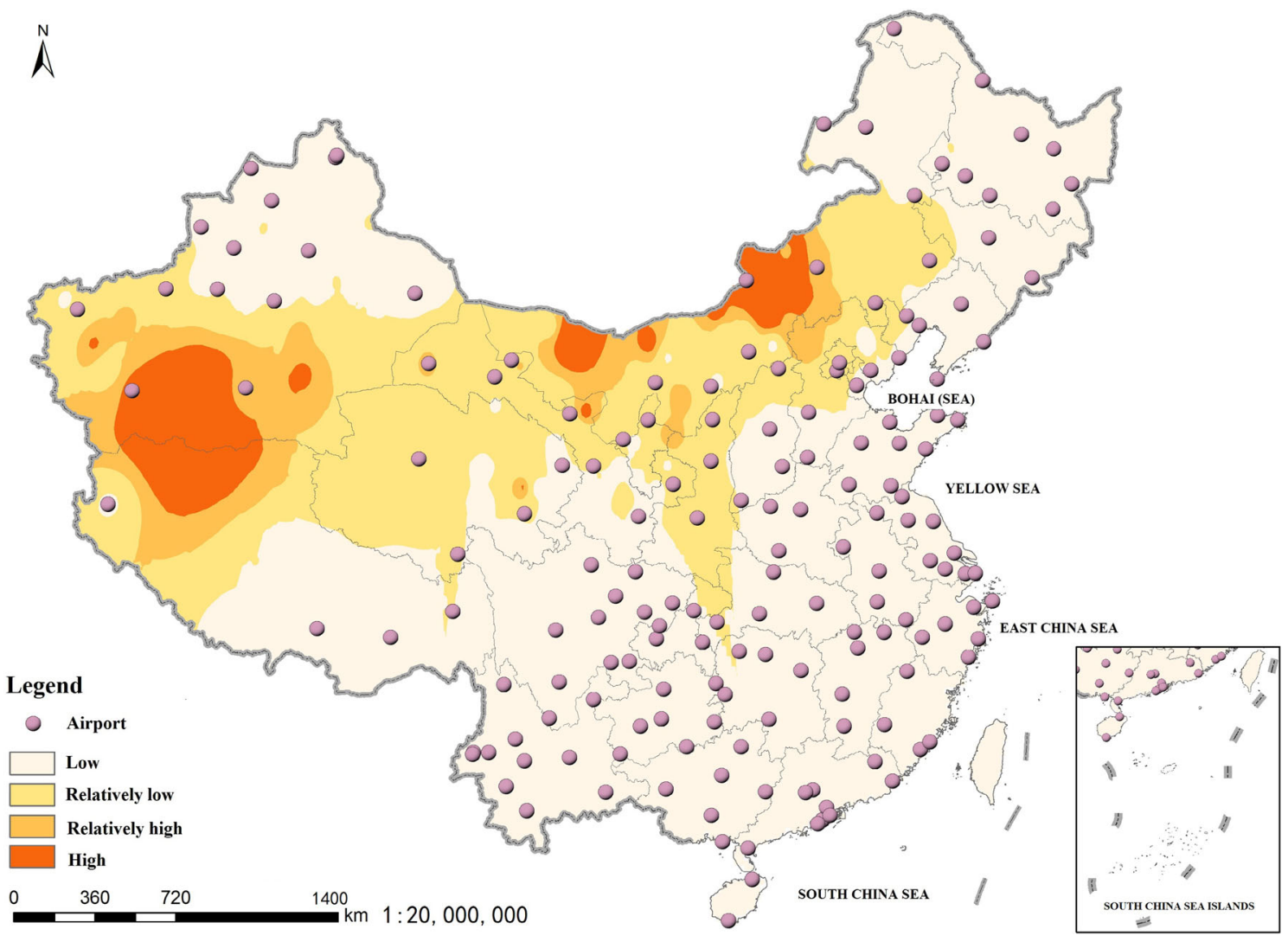

Fig. 7 Spatial distribution of Chinese civil airports and sandstorm occurrence frequencies

\subsection{Results and Analyses}

In order to perform the hazard impact simulation, we first divide the map of China into $20 \times 25$ grids. Grids that are completely within the Chinese territory are assigned a size value of 1 . Grids on the border of the Chinese territory, depending on how much of the grid is within China, take a size value between 0 and 1 . The total area size of the Chinese territory is derived by summing up these size values. The hazard occurrence probability for each grid is determined based on the spatial distribution of random hazards, rainstorms, and sandstorms. The hazard occurrence probability of random hazards is the same for all grids. For any specific percentage of hazard covered area, we can calculate the number of grids needed to make up such a percentage, and then randomly choose the appropriate number of grids as the hazard covered areas. Finally, we calculate the percentage of effectively impacted nodes by hazards through counting the number of airports in the hazard covered grids.

In this article, for each test case, we change the percentage of hazard covered area from 0 to $100 \%$ by a step of $0.5 \%$. For each given percentage of hazard covered area, we conduct 100 hazard simulation tests. The simulation results are given in Fig. 8. The SVI values of the
Chinese civil aviation network system are presented in Table 1, in which lower ASVI/RSVI values mean higher robustness of the Chinese civil aviation network system to the hazards, and vice versa. When the ASVI/RSVI values are close to zero, the system behaves neutrally to the hazards.

In scenario 1 where hazards have a uniform spatial distribution, if we only consider airports directly impacted by the hazards (that is, airports in the area covered by the hazards, test case 1) the ASVI is -0.8363 and the RSVI is -1.7384 (Table 1). Both indices have negative values, and the associated impact curve is slightly below the neutral line (Fig. 8a). This indicates that the Chinese civil aviation network system is slightly robust to spatially localized hazards with uniform distribution. The reason is that most Chinese airports are located in the southeastern area of the country, that is, the spatial distribution of airports is not uniform in reality (Figs. 6, 7). Therefore, a hazard with a uniform spatial distribution will impact fewer airports when compared with the neutral line (the impact curve when both hazards and airports follow a uniform distribution).

As explained in Sect. 2, in a network system, the impact of a spatially localized hazard may spread from covered area to non-covered area. Therefore, to comprehensively 

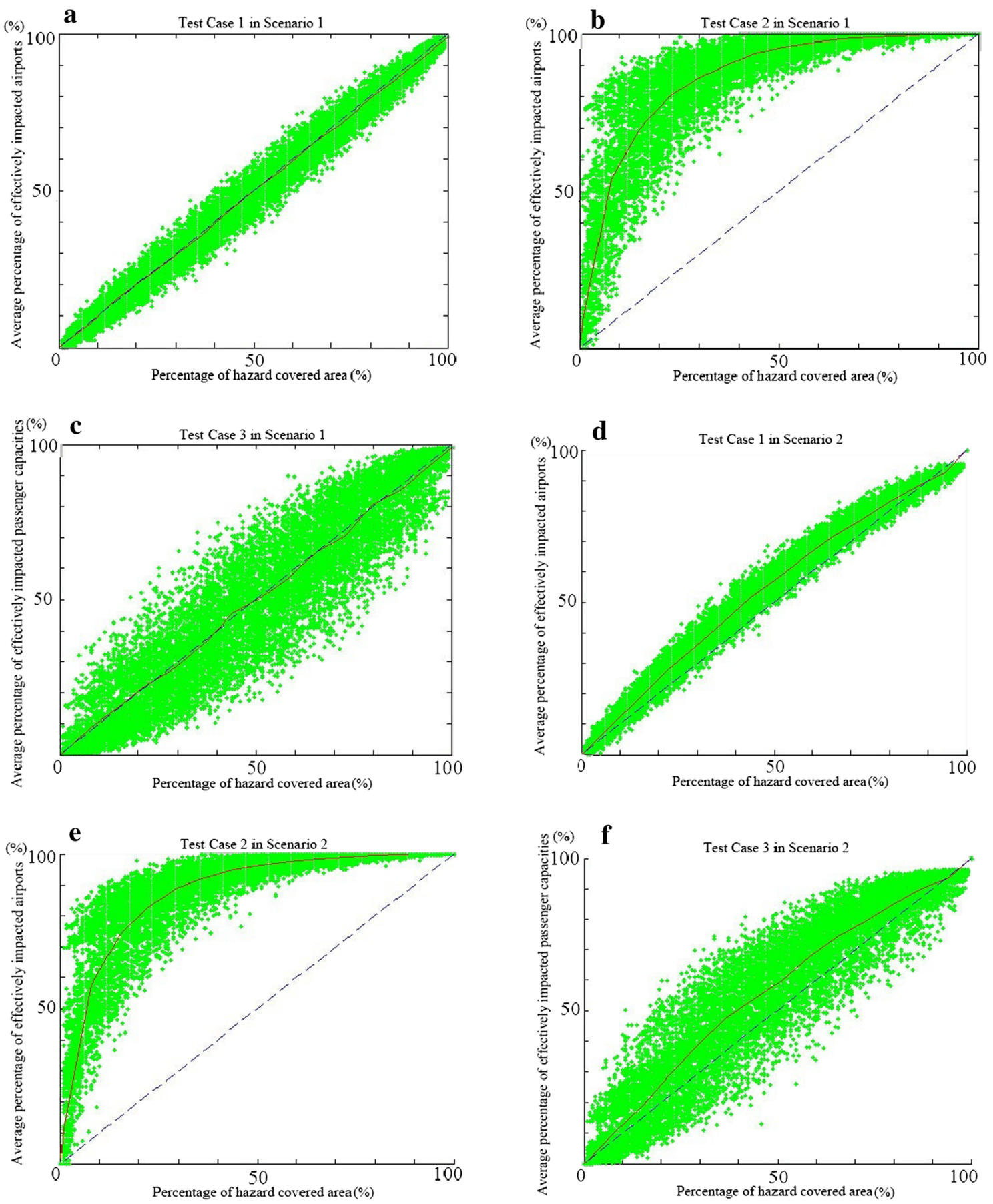

Fig. 8 Simulation results on the spatial vulnerability of the Chinese civil aviation network system (a-i)

assess the performance (spatial vulnerability in this study) of a network system, we need to take into account the impact of hazards on those airports that are not directly covered by the hazards. In test case 2 of scenario 1 we consider not only the directly impacted airports, but also those that have direct flights from/to the directly impacted airports. In this case, both the ASVI and the RSVI become positive (Table 1), and the impact curve is largely above the neutral line (Fig. 8b). This indicates that, when considering connections between airports, the Chinese civil aviation network system is highly vulnerable to spatially localized hazards, even though the spatial distribution of hazards is uniform, and that of airports, is not uniform.

Also as discussed in Sect. 2, the impact of hazards on a network system can be evaluated using different indicators. The number of effectively impacted airports can be used to 

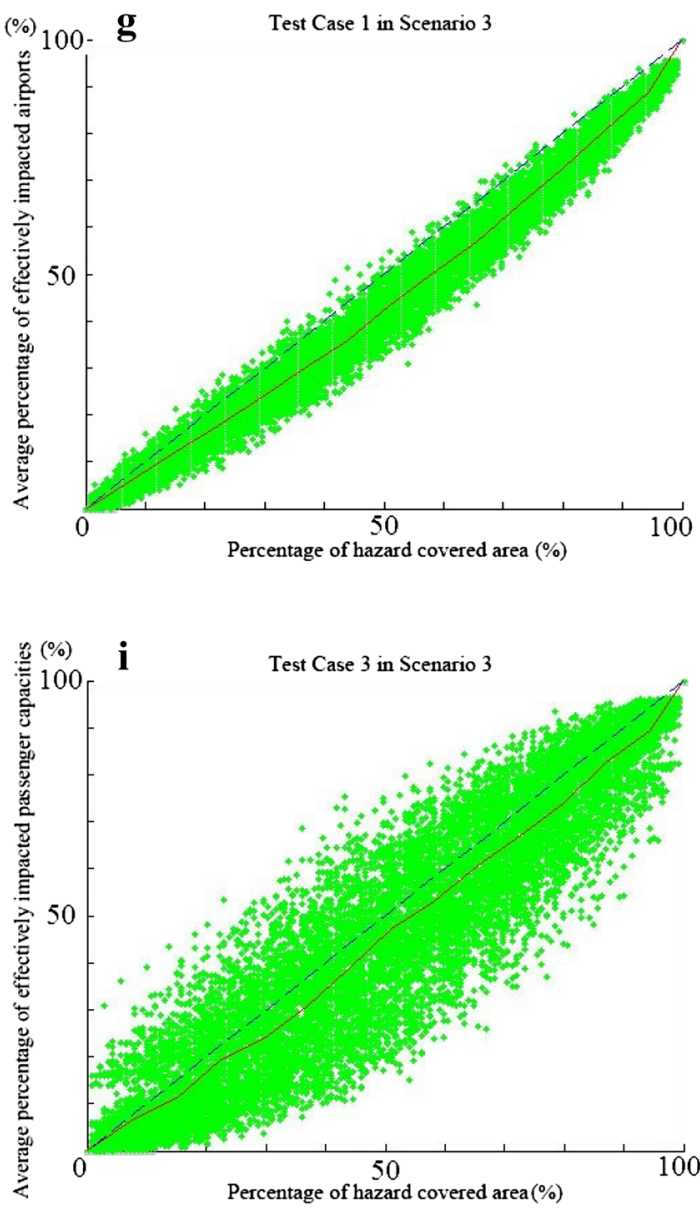

Fig. 8 continued

quantify the impact of hazards. Likewise, daily passenger capacity losses of airports in the system can also signify the impact of hazards. An airport with a larger passenger capacity is clearly more important than an airport with a smaller capacity in the civil aviation network system. Thus, in test case 3 of scenario 1, we consider the passenger capacity at the directly impacted airports, that is, when plotting the impact curve, we replace effectively impacted airports with effectively impacted passenger capacities. The result shows that, by including the passenger capacity consideration, the Chinese civil aviation network system is still slightly robust to spatially localized hazards with uniform distribution. The main reason is that most economic activities, which demand larger transportation

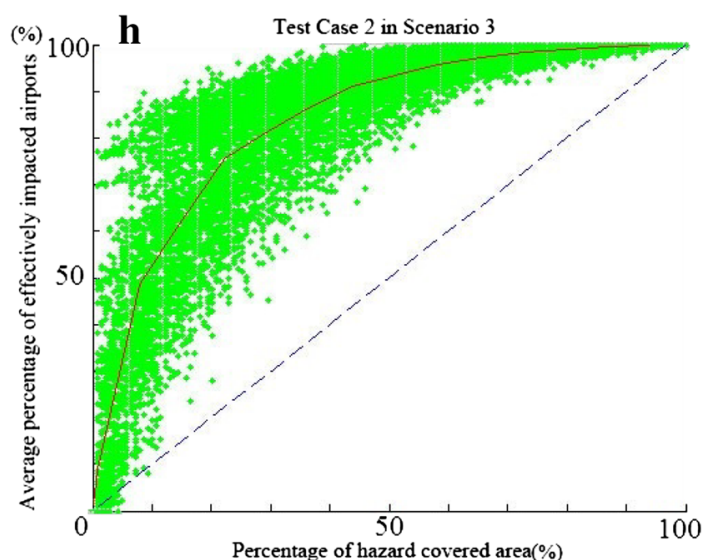

capacities, including airport capacities, are concentrated in the southeastern area of China. In other words, the spatial distribution of airport passenger capacities is not uniform in reality. Therefore, in terms of directly impacted passenger capacities, the system is robust to spatially localized hazards with a uniform distribution.

In scenario 2 and scenario 3, we conduct simulation studies on hazards with non-uniform distribution. In scenario 2, the occurrence probability of hazards is determined by the spatial distribution of rainstorms occurrences in China (Fig. 6), that is, the higher rainstorm probability a location has, the greater possibility the hazard may occur. Table 1 and Fig. 8d-f clearly show that, in all the three test cases of scenario 2, the ASVI and RSVI values are

Table 1 Spatial vulnerability index values of the Chinese civil aviation network system

\begin{tabular}{|c|c|c|c|c|c|c|}
\hline & \multicolumn{2}{|c|}{ Test case 1} & \multicolumn{2}{|c|}{ Test case 2} & \multicolumn{2}{|c|}{ Test case 3} \\
\hline & $V_{\mathrm{ASVI}}$ & $V_{\mathrm{RSVI}}$ & $V_{\mathrm{ASVI}}$ & $V_{\mathrm{RSVI}}$ & $V_{\text {ASVI }}$ & $V_{\mathrm{RSVI}}$ \\
\hline Scenario 1 & -0.8363 & -1.7384 & 50.6176 & 247.9066 & -1.1446 & -3.0734 \\
\hline Scenario 2 & 5.6979 & 18.7048 & 53.1297 & 276.8941 & 8.2663 & 25.2023 \\
\hline Scenario 3 & -8.4845 & -21.4787 & 47.3522 & 226.0798 & -6.3092 & -16.0187 \\
\hline
\end{tabular}


positive, which indicate the Chinese civil aviation network system is highly vulnerable to rainstorms. The explanation is that the spatial distribution of rainstorms is largely consistent with that of airports in China (Fig. 6), that is, the airports are concentrated in the areas with high frequency of rainstorms. Sufficient rainfall is associated with higher agriculture production, which is in turn crucial to supporting other economic activities that have a demand for airports. If a hazard often occurs in areas with dense airports, it will certainly cause severe disruption to the system.

In scenario 3, when hazard spatial distribution is determined by sandstorm occurrences (Fig. 7), the vulnerability assessment results are very different from scenario 2 . Specifically, ASVI and RSVI values in all three test cases of scenario 3 are less than that in scenario 2 . In test case 1 and test case 3 of scenario 3 , the simulation results show that the Chinese civil aviation network system is robust to sandstorms. On the contrary, in test case 1 and test case 3 of scenario 2, the system is vulnerable to rainstorms. This is because the spatial distribution of sandstorms in China is not consistent with the distribution of airports (see Fig. 7). Frequent severe sandstorms often happen in areas with sparse airports. Therefore, the Chinese civil aviation network system is robust to sandstorms.

Furthermore, in any scenario, when compared with either test case 1 or test case 3, test case 2 has much higher ASVI and RSVI values. These results suggest that, when taking connections between airports into consideration, the Chinese civil aviation network system is vulnerable to spatially localized hazards, no matter the spatial distribution of hazards is uniform or non-uniform. Meanwhile, the results also imply that the consideration of connections between nodes for the spreading impact of hazards makes a great difference and therefore is crucial for studying the performance of a network system in the face of spatially localized hazards.

\section{Conclusions and Future Work}

Inspired by Wilkinson's study on spatial vulnerability of the European air traffic network in 2012, this article further develops a quantitative model to study spatial vulnerability of civil aviation network systems under the impact of spatially localized hazards. This model considers hazard location and hazard covered area, as well as the spatially spreading impact of hazards, and proposes two spatial vulnerability indices-the ASVI and the RSVI-to quantitatively assess the vulnerability of network systems in the face of spatially localized hazards.

We apply the spatial vulnerability model to study the Chinese civil aviation network system under three specific hazard scenarios. The results show that the proposed spatial vulnerability model is effective and useful to study civil aviation network systems as conclusions are well in line with the general situation of the studied system.

Extensive efforts are still required to explore the full potential of the proposed spatial vulnerability model. Some directions for future research include: (1) conduct further theoretical modification, extension, and analyses of the proposed spatial vulnerability model, for instance, by considering the preparedness level against hazards at all nodes ( $\mathrm{Li}$ et al. 2015) because a node's preparedness level against hazards plays an important role in the spatial vulnerability of a network system; consider hazard duration; investigate how to calculate the indirect impact of spatially localized hazards more precisely. (2) Study civil aviation systems in more depth, for example, by collecting more comprehensive data of airports and flights worldwide and comparing spatial vulnerability of these systems in different countries. (3) Apply the proposed spatial vulnerability model to study other network systems, such as communication, energy, and other transportation systems.

Acknowledgments This work was supported in part by the National Basic Research Program of China (Grant No. 2012CB955404), the National Natural Science Foundation of China (Grant No. 61472041), and the Foundation for Innovative Research Groups of the National Natural Science Foundation of China (Grant No. 41321001). Some preliminary results of this study were presented at the 2014 IEEE 17th International Conference on Intelligent Transportation Systems (ITSC 2014), 8-11 October 2014, Qingdao, China.

Open Access This article is distributed under the terms of the Creative Commons Attribution 4.0 International License (http://crea tivecommons.org/licenses/by/4.0/), which permits unrestricted use, distribution, and reproduction in any medium, provided you give appropriate credit to the original author(s) and the source, provide a link to the Creative Commons license, and indicate if changes were made.

\section{References}

Albert, R., H. Jeong, and A.N. Barabasi. 2000. Error and attack tolerance of complex networks. Nature 406: 378-382.

Arulselvan, A., C.W. Commander, L. Elefteriadou, and P.M. Pardalos. 2009. Detecting critical nodes in sparse graphs. Computers \& Operations Research 36(7): 2193-2200.

Burghouwt, G., J. Hakfoort, and J.R. van Eck. 2003. The spatial configuration of airline networks in Europe. Air Transport Management 9(5): 309-323.

Chi, L.P., R. Wang, H. Su, and X. Cai. 2003. Structural properties of US flight network. Chinese Physics Letters 20(8): 1393-1396.

Chinese Meteorological Data Services. n.d. http://www.escience.gov. $\mathrm{cn} /$ metdata/page/index.html. Accessed 1 May 2014.

Church, R.L., M.P. Scaparra, and R.S. Middleton. 2004. Identifying critical infrastructure: The median and covering facility interdiction problems. Annals of the Association of American Geographers 94(3): 491-502. 
Church, R.L., and M.P. Scaparra. 2007. Protecting critical assets: The r-interdiction median problem with fortification. Geographical Analysis 39(2): 129-146.

Corley, H.W., and D.Y. Sha. 1982. Most vital links and nodes in weighted networks. Operations Research Letters 1(4): 157-160.

Cutter, S.L., B.J. Boruff, and W.L. Shirley. 2003. Social vulnerability to environmental hazards. Social Science Quarterly 84(2): 242-261.

Ding, M.T., and C. Miao. 2014. GIS-based risk assessment of landslide hazards in Lushan earthquake-stricken areas. Journal of Natural Disasters 23(4): 81-90.

Gastner, M.T., and M.E.J. Newman. 2006. The spatial structure of networks. The European Physical Journal B 49(2): 247-252.

Ghare, P.M., D.C. Montgomery, and W.C. Turner. 1971. Optimal interdiction policy for a flow network. Naval Research Logistics Quarterly 18(1): 37-45.

Grubesic, T.H., M.E. O'Kelly, and A.T. Murray. 2003. A geographic perspective on commercial internet survivability. Telematics and Informatics 20(1): 51-69.

Grubesic, T.H., and A.T. Murray. 2006. Vital nodes, interconnected infrastructures and the geographies of network reliability. Annals of the Association of American Geographers 96(1): 64-83.

Guimera, R., and L.A.N. Amaral. 2004. Modeling the world-wide airport network. The European Physical Journal B 38(2): $381-385$

ICAO (International Civil Aviation Organization). 2014. Annual report of the ICAO council: The world of air transport in 2013. http://www.icao.int/annual-report-2014/Pages/CH/default_CH. aspx. Accessed 10 Dec 2014.

IPCC (Intergovernmental Panel on Climate Change). 2001. Climate change 2001: Impact, adaption and vulnerability. Summary for policymakers. http://www.ipcc.ch/pdf/climate-changes-2001/ impact-adaptation-vulnerability/impact-spm-en.pdf. Accessed 10 Sept 2016.

Israeli, E., and R.K. Wood. 2002. Shortest-path network interdiction. Networks 40(2): 97-111.

Jaiswal, K.S., D.J. Wald, P.S. Earle, K.A. Porter, and M. Hearne. 2011. Earthquake casualty models within the USGS prompt assessment of global earthquakes for response (PAGER) system. In Human casualties in earthquakes: Progress in modelling and mitigation, ed. R. Spence, E. So and C. Scawthorn, 83-94. New York: Springer.

Janić, M. 2015. Modelling the resilience, friability and costs of an air transport network affected by a large-scale disruptive event. Transportation Research Part A 71: 1-16.

Li, W., and X. Cai. 2004. Statistical analysis of airport network of China. Physical Review E 69(4): 1-6.

Li, H., X.M. Guo, Z. Xu, and X.B. Hu. 2014. A study on the spatial vulnerability of the civil aviation network system in China. In Proceedings of 17 th international IEEE conference on intelligent transportation systems (ITSC), 8-11 October 2014, Qingdao, China, 2650-2655.

Li, H., X. Hu, X. Guo, and Z. Xu. 2015. Assessment on spatial vulnerability of network systems under spatially local hazards. In
Emerging economies, risk and development, and intelligent technology: Proceedings of the 5th international conference on risk analysis and crisis response, 1-3 June 2015, Tangier, Morocco, ed. C. Huang, A. Lyhyaoui, G. Zhai and N. Benhayoun, 359-368. Boca Raton, FL: CRC Press.

Li, H., P.Y. Zhang, and Y.Q. Cheng. 2008. Concepts and assessment methods of vulnerability. Progress in Geography 27(2): 18-25.

Lim, C., and J.C. Smith. 2007. Algorithms for discrete and continuous multicommodity flow network interdiction problems. IIE Transactions 39(1): 15-26.

Mazzocchi, M., F. Hansstein, and M. Ragona. 2010. The 2010 volcanic ash cloud and its financial impact on the European airline industry. CESifo Forum, 11(2), 92-100.

Murray, A.T., and T. Grubesic. 2007. Reliability and vulnerability in critical infrastructure: A quantitative geographic perspective. Heidelberg: Springer.

Murray, A.T., T.C. Matisziw, and T.H. Grubesic. 2007. Critical network infrastructure analysis: Interdiction and system flow. Journal of Geographical Systems 9(2): 103-117.

Myung, Y.S., and H.J. Kim. 2004. A cutting plane algorithm for computing k-edge survivability of a network. European Journal of Operational Research 156(3): 579-589.

Omidvar, B., B. Gatmiri, and S. Derakhshan. 2012. Experimental vulnerability curves for the residential buildings of Iran. Natural Hazards 60(2): 345-365.

Papathoma-Köhle, M., M.K.R. Totschnig, T. Glade. 2012. Improvement of vulnerability curves using data from extreme events: Debris flow event in South Tyrol. Natural Hazards 64(3): 2083-2105.

Qunar. 2014. http://www.qunar.com/?tab=hotel\&ex_track=auto_ 4e0d874a. Accessed 1 Sept 2014.

Shi, P.J., N. Li, Q. Ye, W. Dong, G. Han, and W. Fang. 2009. Research on global environment change and integrated disaster risk governance. Advances in Earth Science 24(4): 428-435 (in Chinese).

Tran, L.T., R.V. O'Neil, and R. Smith. 2010. Spatial pattern of environmental vulnerability in the Mid-Atlantic region, USA. Applied Geography 30(2): 191-202.

Turner, B.L., R.E. Kasperson, A.M. Pamela, J.J. McCarthy, R.W. Corell, L. Christensen, N. Eckley, J.X. Kasperson, A. Luers, M.L. Martello, C. Polsky, A. Pulsipher, and A. Schiller. 2003. A framework for vulnerability analysis in sustainability science. Proceedings of the National Academy of Sciences of the United States of America 100(14): 8074-8079.

Wilkinson, S.M., D. Sarah, and M. Shu. 2012. The vulnerability of the European air traffic network to spatial hazards. Natural Hazards 60(3): 1027-1036.

Wollmer, R. 1964. Removing arcs from a network. Operation Research 12(6): 934-940.

Wood, R.K. 1993. Deterministic network interdiction. Mathematical and Computer Modeling 17(2): 1-18.

Zhou, Y., N. Li, W. Wu, J. Wu, and P. Shi. 2014. Local spatial and temporal factors influencing population and societal vulnerability to natural disasters. Risk Analysis 34(4): 614-639. 